\title{
Survey on attitudes towards COVID-19 preventive measures and protective behavior in Slovenia
}

Anže Mihelič, University of Maribor Faculty of Criminal Justice and Security

Luka Jelovčan, University of Maribor Faculty of Criminal Justice and Security

Kaja Prislan, University of Maribor Faculty of Criminal Justice and Security

\begin{abstract}
This paper presents the dataset underlying the research on attitudes towards and compliance with instructed preventive measures during the COVID-19 pandemic in Slovenia. A summary of results and questionnaires in English and Slovenian language are provided. The study was conducted at the beginning of the first pandemic wave when citizens' uncertainty about the disease was at its peak.
\end{abstract}

Keywords and Phrases: epidemic, pandemic, COVID-19, general deterrence theory, protection-motivation theory

\section{INTRODUCTION}

The year 2020 was severely marked by the COVID-19 pandemic. The health crisis was accompanied by several restrictive measures (such as restrictions on free movement within the country, closing the national borders, and stopping the work processes in industries) enforced and controlled by the authorities. A general deterrence theory is applicable for investigating citizens' compliance in situations such as the one mentioned - when offenders are punished for non-compliance. This paper presents the research in which factors stemming from general deterrence theory is compared to the factors related to the protection-motivation theory, a generally accepted theory for explaining protective behavior.

The paper is structured as follows. In the next section, we provide details of the method. Section 3 describes the results. In the final section, the results are discussed.

\section{METHOD}

A survey questionnaire was designed to measure the general deterrence theory constructs (perceived severity of the formal and informal punishment and perceived certainty of formal and informal punishment (Allen et al., 2017; Moody et al., 2018)) and protection-motivation theory constructs (perceived vulnerability (Ho, 2012; Myers \& Goodwin, 2011), perceived severity (Myers \& Goodwin, 2011), self-efficacy, and response efficacy (Milne et al., 2002; Thrasher et al., 2016)). The dependent variable was self-reported compliance with instructed measures (items were self-developed). We also investigated attitudes towards these preventive measures. Items for attitudes were adapted from (Zhang et al., 2019). Altogether the questionnaire included ten constructs and 35 items. The questionnaire was originally developed in English and translated to the Slovenian language. The attitude was measured on a five-point semantic differential scale. Informal severity was measured on a five-point Likert-type scale from 1 - Not ashamed at all to 5 - Extremely ashamed (sl. 1 - Sploh ne to 5 Izjemno močno). Other items were measured on a five-point Likert scale from 1 - Strongly disagree to 5 - 
Strongly agree (sl. 1 - Sploh se ne strinjam to 5 - Popolnoma se strinjam). The questionnaire items are presented in Table 1 (see Appendix A: Tables).

\subsection{Data collection}

The online survey was conducted between April and May 2020 and distributed in ten Facebook groups to provide sample diversity. In total, we collected $N=401$ responses. After excluding inadequate cases, we ended up with $N=394$ respondents. The sample consisted of 76 percent women with a mean age of 31.3 years. Nearly half (45.7 percent) of respondents had less than bachelor's degree, 37.7 percent had bachelor's degree, 15.4 percent completed master's degree, while 1.3. percent hold a PhD. More than half (51.2 percent) of respondents were employed or self-employed, 39.3 percent were students, while 8.0 percent were unemployed or retired.

\section{RESULTS}

The frequencies of respondent's answers to each item are presented in Table 2 (see Appendix A: Tables).

\section{DISCUSSION}

The research has shown that respondents did not fear being infected by the virus SARS-CoV-2 and mainly were confident that they are self-efficient in protecting against the disease. They deemed the measures as efficient and had somewhat favorable attitudes towards instructed measures. Regarding the punishment, the informal severity was perceived as highest and formal certainty as lowest. They mostly followed the recommended preventive measures. Such compliance is - to some extent - to be expected since, at the beginning of the pandemic, the requirements for entering public and other facilities were highly supervised. The uncertainty of the conditions also shows through the high share of missing values at the attitudes.

\section{LITERATURE}

Allen, S., Murphy, K., \& Bates, L. (2017). What drives compliance? The effect of deterrence and shame emotions on young drivers' compliance with road laws. Policing and Society, 27(8), 884-898. https://doi.org/10.1080/10439463.2015.1115502

Ho, H. S. (2012). Use of face masks in a primary care outpatient setting in Hong Kong: Knowledge, attitudes and practices. Public Health, 126(12), 1001-1006. https://doi.org/10.1016/j.puhe.2012.09.010

Milne, S., Orbell, S., \& Sheeran, P. (2002). Combining motivational and volitional interventions to promote exercise participation: Protection motivation theory and implementation intentions. British Journal of Health Psychology, 7(2), 163-184. https://doi.org/10.1348/135910702169420

Moody, G. D., Siponen, M., \& Pahnila, S. (2018). Toward a unified model of information security policy compliance. MIS Quarterly, 42(1), 285-311. https://doi.org/10.25300/MISQ/2018/13853

Myers, L. B., \& Goodwin, R. (2011). Determinants of adults' intention to vaccinate against pandemic swine flu. BMC Public Health, 11(August 2009), 1-8. https://doi.org/10.1186/1471-2458-11-15

Thrasher, J. F., Swayampakala, K., Borland, R., Nagelhout, G., Yong, H. H., Hammond, D., Bansal-Travers, M., Thompson, M., \& Hardin, J. (2016). Influences of Self-Efficacy, Response Efficacy, and Reactance on Responses to Cigarette Health Warnings: A Longitudinal Study of Adult Smokers in Australia and Canada. Health Communication, 31(12), 1517-1526. https://doi.org/10.1080/10410236.2015.1089456

Zhang, C. Q., Chung, P. K., Liu, J. D., Chan, D. K. C., Hagger, M. S., \& Hamilton, K. (2019). Health Beliefs of 
Wearing Facemasks for Influenza A/H1N1 Prevention: A Qualitative Investigation of Hong Kong Older Adults. Asia-Pacific Journal of Public Health, 31(3), 246-256.

https://doi.org/10.1177/1010539519844082 
APPENDIX A: Tables

Table 1: Questionnaire items in Slovene and English

\begin{tabular}{|c|c|c|c|}
\hline Construct & Code & Item in Slovene & Item in English \\
\hline \multirow[t]{8}{*}{$\overline{\text { Attitude }}$} & A1 & $\begin{array}{l}\text { Menim, da so priporočena zaščitna navodila, dana s } \\
\text { strani vlade in zdravstvenih ustanov: } \\
\text { škodljiva - - - - - koristna. }\end{array}$ & $\begin{array}{l}\text { I think recommended protective instructions given } \\
\text { by government and health institutions are: harmful } \\
\end{array}$ \\
\hline & $\mathrm{A} 2$ & $\begin{array}{l}\text { Menim, da so priporočena zaščitna navodila, dana s } \\
\text { strani vlade in zdravstvenih ustanov: } \\
\text { nezaželena - - - - - zaželena. }\end{array}$ & $\begin{array}{l}\text { I think recommended protective instructions given } \\
\text { by government and health institutions are: } \\
\text { undesirable - . - - desirable. }\end{array}$ \\
\hline & A3 & $\begin{array}{l}\text { Menim, da so priporočena zaščitna navodila, dana s } \\
\text { strani vlade in zdravstvenih ustanov: } \\
\text { slaba - - - - dobra. }\end{array}$ & $\begin{array}{l}\text { I think recommended protective instructions given } \\
\text { by government and health institutions are: bad - - } \\
\text { - - good. }\end{array}$ \\
\hline & A4 & $\begin{array}{l}\text { Menim, da so priporočena zaščitna navodila, dana s } \\
\text { strani vlade in zdravstvenih ustanov: } \\
\text { omejevalna - - - - neomejevalna. }\end{array}$ & $\begin{array}{l}\text { I think recommended protective instructions given } \\
\text { by government and health institutions are: } \\
\text { restrictive - . - - unrestrictive. }\end{array}$ \\
\hline & A5 & $\begin{array}{l}\text { Menim, da so priporočena zaščitna navodila, dana s } \\
\text { strani vlade in zdravstvenih ustanov: } \\
\text { neugodna - - - - ugodna. }\end{array}$ & $\begin{array}{l}\text { I think recommended protective instructions given } \\
\text { by government and health institutions are: } \\
\text { unfavorable - . - - favorable. }\end{array}$ \\
\hline & A6 & $\begin{array}{l}\text { Menim, da so priporočena zaščitna navodila, dana s } \\
\text { strani vlade in zdravstvenih ustanov: } \\
\text { nepravična - - - - - pravična. }\end{array}$ & $\begin{array}{l}\text { I think recommended protective instructions given } \\
\text { by government and health institutions are: unfair - } \\
\text { - - - fair. }\end{array}$ \\
\hline & A7 & $\begin{array}{l}\text { Menim, da so priporočena zaščitna navodila, dana s } \\
\text { strani vlade in zdravstvenih ustanov: } \\
\text { nerazumna - - - - razumna. }\end{array}$ & $\begin{array}{l}\text { I think recommended protective instructions given } \\
\text { by government and health institutions are: } \\
\text { irrational - . - - rational. }\end{array}$ \\
\hline & A8 & $\begin{array}{l}\text { Menim, da so priporočena zaščitna navodila, dana s } \\
\text { strani vlade in zdravstvenih ustanov: } \\
\text { nepotrebna - - - - - potrebna. }\end{array}$ & $\begin{array}{l}\text { I think recommended protective instructions given } \\
\text { by government and health institutions are: } \\
\text { unnecessary - - - - - necessary. }\end{array}$ \\
\hline \multirow[t]{3}{*}{$\begin{array}{l}\text { Protective } \\
\text { behavior }\end{array}$} & PB1 & $\begin{array}{l}\text { Sledim navodilom o samozaščitnih ukrepih, ki jih je } \\
\text { podala medicinska stroka. }\end{array}$ & $\begin{array}{l}\text { I am following the instructions regarding self- } \\
\text { protective actions given by medical professionals. }\end{array}$ \\
\hline & PB2 & $\begin{array}{l}\text { Sledim navodilom o samozaščitnih ukrepih, ki jih je } \\
\text { podala vlada. }\end{array}$ & $\begin{array}{l}\text { I am following the instructions regarding self- } \\
\text { protective actions given by the authorities. }\end{array}$ \\
\hline & PB3 & $\begin{array}{l}\text { Sledim navodilom o samozaščitnih ukrepih, ki so jih } \\
\text { podale mednarodne medicinske in zdravstvene } \\
\text { organizacije. }\end{array}$ & $\begin{array}{l}\text { I am following the instructions regarding self- } \\
\text { protective actions given by international medical } \\
\text { and health organisations. }\end{array}$ \\
\hline \multirow[t]{3}{*}{$\begin{array}{l}\text { Perceived } \\
\text { vulnerability }\end{array}$} & PV1 & $\begin{array}{l}\text { Obstaja visoka možnost, da se bom v naslednjih tednih } \\
\text { okužil s COVID-19. }\end{array}$ & There is a high chance of me getting COVID-19. \\
\hline & PV2 & Možnost okužbe s COVID-19 je zame zelo realna. & $\begin{array}{l}\text { Getting COVID-19 is currently a high possibility } \\
\text { for me. }\end{array}$ \\
\hline & PV3 & Počutim se ranljivega za COVID-19. & I feel vulnerable to COVID- 19. \\
\hline \multirow{3}{*}{$\begin{array}{l}\text { Perceived } \\
\text { severity }\end{array}$} & PS1 & Zdravstveni zapleti povezani s COVID-19 so resni. & Complications from COVID-19 are serious. \\
\hline & PS2 & Če oseba dobi COVID-19, lahko resno zboli. & $\begin{array}{l}\text { If a person gets a COVID-19, he/she could get } \\
\text { very sick. }\end{array}$ \\
\hline & PS3 & COVID-19 je nevarna bolezen. & COVID-19 is a dangerous disease. \\
\hline \multirow[t]{2}{*}{$\begin{array}{l}\text { Self- } \\
\text { efficacy }\end{array}$} & SE1 & $\begin{array}{l}\text { Prepričan sem v svojo sposobnost zaščite pred COVID- } \\
19 .\end{array}$ & $\begin{array}{l}\text { I feel confident in my ability to protect myself from } \\
\text { COVID-19. }\end{array}$ \\
\hline & $\begin{array}{l}\text { SE2 } \\
\text { SE3 }\end{array}$ & $\begin{array}{l}\text { Ni se težko zaščititi pred COVID-19. } \\
\text { Samozaščita pred virusom COVID-19 je preprosta. }\end{array}$ & $\begin{array}{l}\text { It is not difficult to protect myself from COVID- } 19 \text {. } \\
\text { Protecting myself from COVID-19 is easy for me. }\end{array}$ \\
\hline \multirow[t]{3}{*}{$\begin{array}{l}\text { Response } \\
\text { efficacy }\end{array}$} & RE1 & $\begin{array}{l}\text { Samozaščitni ukrepi so dober način za zmanjšanje } \\
\text { tveganja, da dobim COVID-19. }\end{array}$ & $\begin{array}{l}\text { Self-protective actions are a good way of reducing } \\
\text { the risk of me getting COVID-19. }\end{array}$ \\
\hline & RE2 & $\begin{array}{l}\text { Če bi deloval samozaščitno, bi zmanjšal tveganje, da } \\
\text { dobim COVID-19. }\end{array}$ & $\begin{array}{l}\text { If I were to take self-protective actions, my } \\
\text { chances of getting COVID-19 would be lower. }\end{array}$ \\
\hline & RE3 & $\begin{array}{l}\text { Samozaščitni ukrepi so učinkovit način za zmanjšanje } \\
\text { moje ranljivosti za okužbo s COVID-19. }\end{array}$ & $\begin{array}{l}\text { The self-protective actions are an efficient way of } \\
\text { reducing my vulnerability to COVID- } 19 \text {. }\end{array}$ \\
\hline
\end{tabular}




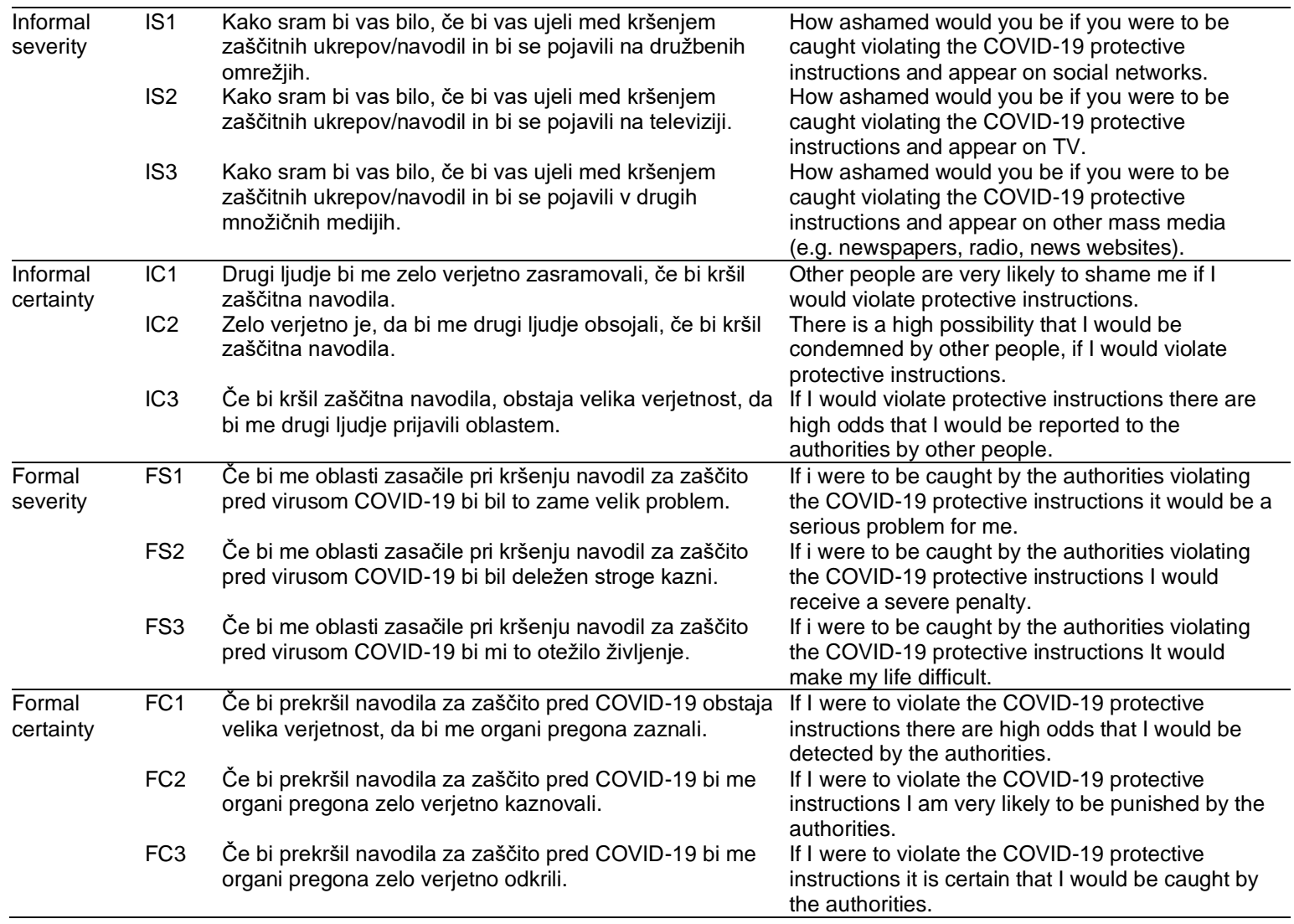


Table 2: Frequencies of answers for each item

\begin{tabular}{|c|c|c|c|c|c|c|}
\hline Item code & Missing & 1 & 2 & 3 & 4 & 5 \\
\hline$\overline{\mathrm{A} 1}$ & 14 & 15 & 30 & 88 & 119 & 128 \\
\hline A2 & 22 & 31 & 65 & 111 & 85 & 80 \\
\hline A3 & 22 & 24 & 45 & 108 & 104 & 91 \\
\hline A4 & 22 & 97 & 59 & 74 & 82 & 60 \\
\hline A5 & 21 & 64 & 89 & 122 & 64 & 34 \\
\hline A6 & 19 & 51 & 73 & 106 & 90 & 55 \\
\hline A7 & 18 & 51 & 59 & 95 & 93 & 78 \\
\hline A8 & 17 & 27 & 57 & 79 & 97 & 117 \\
\hline PB1 & 1 & 10 & 6 & 37 & 132 & 208 \\
\hline PB2 & 2 & 24 & 36 & 85 & 735 & 112 \\
\hline PB3 & 2 & 13 & 11 & 76 & 137 & 155 \\
\hline PV1 & 0 & 111 & 114 & 124 & 32 & 13 \\
\hline PV2 & 0 & 85 & 118 & 96 & 68 & 27 \\
\hline PV3 & 0 & 121 & 120 & 76 & 51 & 26 \\
\hline PS1 & 3 & 19 & 35 & 78 & 154 & 105 \\
\hline PS2 & 2 & 19 & 36 & 67 & 151 & 119 \\
\hline PS3 & 3 & 22 & 49 & 104 & 123 & 93 \\
\hline SE1 & 3 & 2 & 16 & 77 & 198 & 98 \\
\hline SE2 & 1 & 10 & 65 & 134 & 128 & 56 \\
\hline SE3 & 0 & 22 & 62 & 133 & 121 & 56 \\
\hline RE1 & 0 & 7 & 13 & 60 & 185 & 129 \\
\hline RE2 & 0 & 12 & 11 & 73 & 173 & 125 \\
\hline RE3 & 2 & 16 & 28 & 66 & 166 & 116 \\
\hline IS1 & 2 & 72 & 55 & 86 & 98 & 81 \\
\hline IS2 & 2 & 64 & 55 & 80 & 102 & 91 \\
\hline IS3 & 1 & 73 & 48 & 87 & 103 & 82 \\
\hline IC1 & 2 & 41 & 74 & 143 & 100 & 34 \\
\hline IC2 & 3 & 16 & 53 & 119 & 156 & 47 \\
\hline IC3 & 3 & 24 & 93 & 136 & 92 & 46 \\
\hline $\mathrm{FS} 1$ & 3 & 33 & 65 & 127 & 120 & 46 \\
\hline FS2 & 3 & 28 & 60 & 111 & 144 & 48 \\
\hline FS3 & 4 & 55 & 80 & 123 & 92 & 40 \\
\hline $\mathrm{FC} 1$ & 4 & 37 & 113 & 138 & 80 & 22 \\
\hline $\mathrm{FC2}$ & 5 & 25 & 79 & 128 & 127 & 30 \\
\hline FC3 & 6 & 47 & 123 & 145 & 57 & 16 \\
\hline
\end{tabular}

